\title{
Bilateral Toric Phakic Intraocular Lens Implantation for Correction of High Myopic Astigmatism in a Patient with Marfan Syndrome with Lens Coloboma: A Case Report
}

\author{
Bhupesh Singh Sourabh Sharma Suchit Dadia Neha Bharti \\ Sudhank Bharti \\ Bharti Eye Foundation and Hospital, New Delhi, India
}

Keywords

Marfan syndrome $\cdot$ Myopia $\cdot$ Phakic intraocular lens

\begin{abstract}
Marfan syndrome (MFS) is known to cause significant refractive error. Treatment options are limited in this condition for correcting refractive error. Clear lens exchange is done in these cases, but complication rates are high. Loss of accommodation is another concern in these young adults. We report toric phakic intraocular lens ( $\mathrm{plOL}$ ) implantation in improving the uncorrected visual acuity (UCVA) in a known case of MFS with lens coloboma. A 22-year-old female patient with MFS with inferior lens coloboma underwent bilateral toric pIOL implantation in the same sitting. Pre- and post-operative UCVA and best-corrected visual acuity were assessed. Central and peripheral vaulting of the $\mathrm{pIOL}$ in relation to the natural lens was also assessed. UCVA improved from 20/500 to 20/20 in the right and 20/550-20/20 in the left eye. Marked central vaulting with partial peripheral vaulting was achieved. There were no postoperative complications. Phakic IOL implantation surgery could be an effective approach to achieve excellent uncorrected refractive outcome in patients with MFS to treat high myopia.
\end{abstract}

\section{Introduction}

Marfan syndrome (MFS) is an autosomal dominant connective tissue disorder, caused by mutations in the fibrillin-1 gene [1]. Many ocular abnormalities are associated with MFS, including retinal detachment, increased axial length and myopia, flattened cornea, iris and 
ciliary muscle hypoplasia, and ectopia lentis [2]. Patients with MFS may have high corneal [3] as well as lenticular astigmatism. Lenticular astigmatism, both regular and oblique, may arise if there is selective damage to the zonules as in subluxated lenses and lens colobomas. Lens colobomas are congenital lens anomaly, rarely seen with MFS. They are not true colobomas as there is no focal absence of a tissue layer. Occasionally, they can be associated with a coloboma of the iris or fundus.

Corneal refractive surgery is relatively contraindicated in MFS patients and they are dependent on spectacles or contact for lifetime [4]. Toric posterior chamber phakic intraocular lens (pIOL) has emerged as an excellent refractive surgery alternative in such patients [4-6]. Herein, we present a case of MFS with bilateral inferior lens coloboma that underwent toric pIOL implantation with an excellent outcome.

\section{Case Report}

A 22-year-old female patient came to department of refractive surgery services seeking a consult for LASIK surgery to get rid of her spectacles. On ocular examination, her uncorrected visual acuity was 20/500 in the right eye and 20/550 in the left eye, and her bestcorrected visual acuity was 20/20 OD (manifest refraction $-14 /-3 \times 5$ ) and 20/20 OS (manifest refraction $-14 /-3 \times 2$ ). There was no change in her refraction since last 2 years. The keratometry readings were as follows: OD K $\mathrm{K}_{1} 41.1 \times 103 / \mathrm{K}_{2} 41.9 \times 13$; OS $\mathrm{K}_{1} 40.6 \times 96 /$ $\mathrm{K}_{2} 41.5 \times 6$ (Pentacam; Oculus, Inc., Lynnwood, WA, USA). Intraocular pressures (IOP) were 15 and 18 in her right and left eyes, respectively.

The patient was explained in detail regarding possibility of pIOL placement and refractive lens exchange (RLE). The patient was elected for placement of toric Implantable Collamer Lens (ICL) in both of her eyes after extensive discussion on the technique, results, and risks of the same.

Slit lamp examination of both eyes revealed a flat cornea with no signs of keratoconus. Corneal topography revealed normal corneal thickness and no signs of corneal ectasia (Pentacam; Oculus, Inc., Lynnwood, WA, USA) (Fig. 1). Dilated slit lamp examination revealed

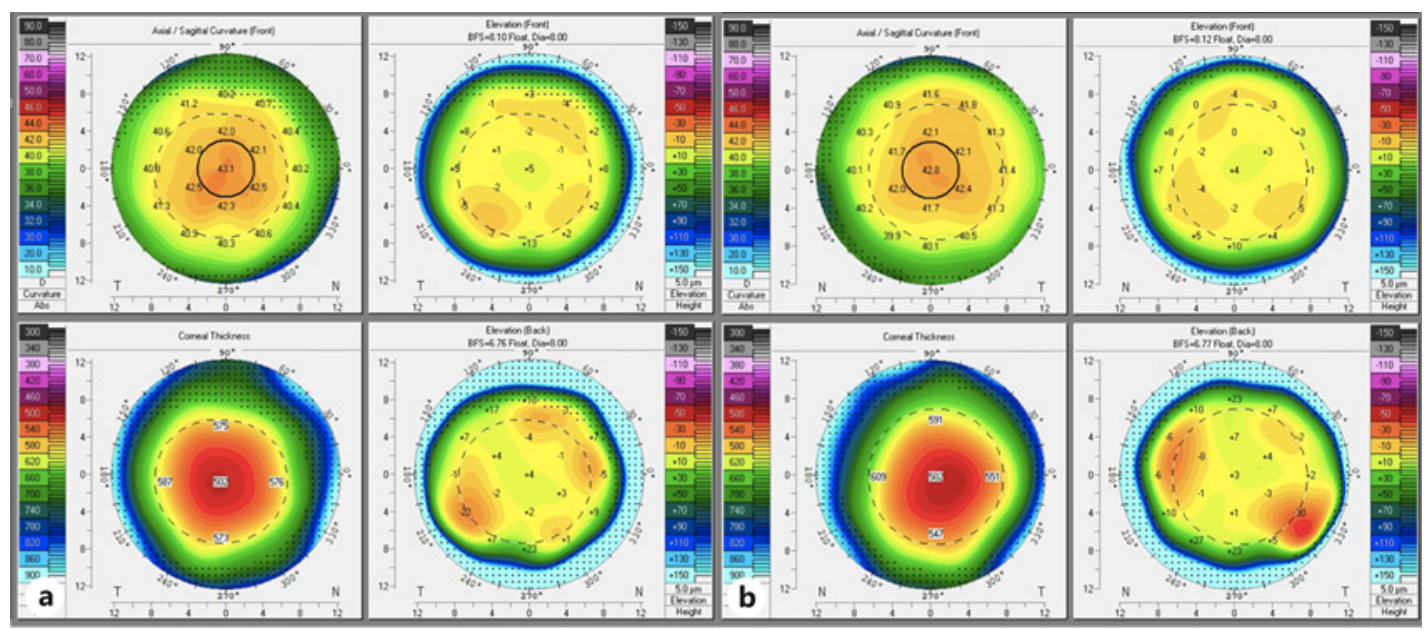

Fig. 1. a Preoperative Scheimpflug corneal topography of the right cornea showing regular against the rule astigmatism with a flat cornea. b Preoperative schiempflug corneal topography of the left cornea showing regular against the rule astigmatism with a flat cornea (note the difference between the topographic cylinder [0.8 and 0.9 D] and manifest refractive cylinder [3 D], indicating presence of significant lenticular astigmatism). 
bilateral inferior lens coloboma. Examination of the posterior segments was notable for myopia bilaterally. The aqueous depth was $3.34 \mathrm{~mm}$ in the right eye and $3.18 \mathrm{~mm}$ in the left eye. The endothelial cell density was 2,600 cells $/ \mathrm{mm}^{2}$ OD and 2,550 cells $/ \mathrm{mm}^{2}$ OS (Specular Microscope CEM-530; NIDEK Co., Aichi, Japan). White to White measurement was done with digital calliper and was measured to be $12.6 \mathrm{~mm}$ in both eyes. She had no other significant ocular history and was not on any topical medications. Systemic examination revealed arachnodactyly, positive wrist and thumb sign, and upper segment to lower segment ratio of $<1.05$. The rest of the systemic work up was normal. Family history was positive (father) for similar ophthalmic manifestations (Fig. 2).

The toric ICL (model V4c; STAAR Surgical, Monrovia, CA, USA) was implanted sequentially in both eyes under topical anaesthesia in the same sitting. The ICL model used was Visian ICL V4c and has been designed with a central hole of $0.36 \mathrm{~mm}$. The toric reference marking in right eye was $0^{\circ}$ and left eye $5^{\circ}$. The incision size was $3.2 \mathrm{~mm}$. We evaluated the subjective refraction, aqueous depth, white to white diameter, keratometry readings, and thinnest pachymetry. These data were sent to Staar ICL company for IOL power calculation. The IOL had a diameter of $12.6 \mathrm{~mm}$ with refractive power of $(-17.5 /+2.5 \times 85)$ and $(-17.5 /+2.5$ $\times 95$ ) for right and left eyes, respectively.

Patient was evaluated on slit lamp 30 min after the procedure and discharged on antibiotics + steroid combination eye drops and preservative-free lubricant eye drops. On postoperative day 1 , the patient's uncorrected visual acuity was $20 / 40$ in both eyes with mild blurring of vision. Her post-operative IOP was 17 in the right and 16 in the left eye. Slit lamp examination showed a well-formed anterior chamber with 1+ cells. The ICL was seen in correct position anterior to the natural crystalline lens with a good vaulting between the 2 . The patient returned 1 week later and reported subjective improvement in her vision, which was noted to be 20/20 in both eyes. Her pIOL vaulting, IOP, and slit lamp examination were unremarkable at all follow-ups (Fig. 3). At last follow-up (18 months post-op), visual acuity was $20 / 20$ in both eyes with adequate ICL vault as measured by anterior segment optical coherence tomography (Fig. 4). The endothelial cell density at 18 months was 2,580 cells/ $\mathrm{mm}^{2}$ OD and 2,514 cells $/ \mathrm{mm}^{2}$ OS.

Fig. 2. Patient with MFS with increased arm span length (a) and arachnodactyly (b). MFS, Marfan syndrome.
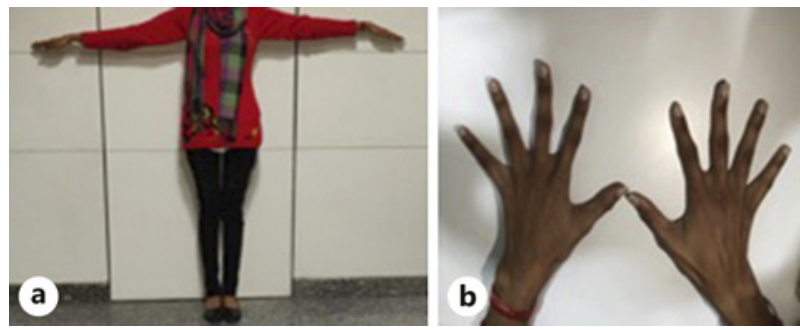

Fig. 3. a One-year post-operative slit lamp photography of the right eye showing the lens-free pupillary area inferiorly with wellcentred ICL. b One-year post-operative slit lamp photography of the left eye showing the lens-free pupillary area inferiorly with wellcentred ICL. A typical coloboma notch is seen inferiorly at lens equator in both eyes. ICL, Implantable Collamer Lens.

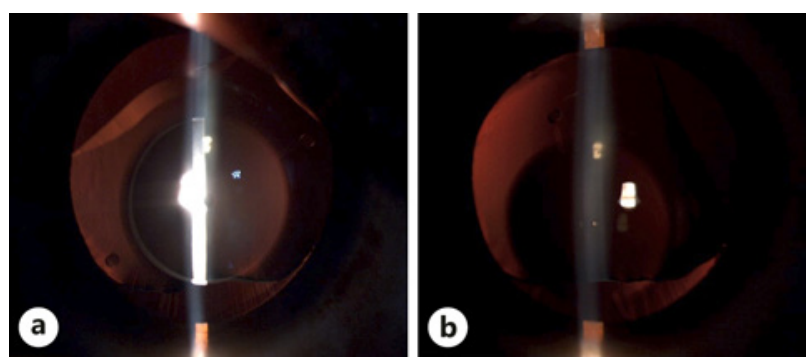


Fig. 4. a One-year ASOCT image of both eyes (right eye (a), left eye (b)) showing ICL vaulting. ASOCT, anterior segment optical coherence tomography; ICL, Implantable Collamer Lens.

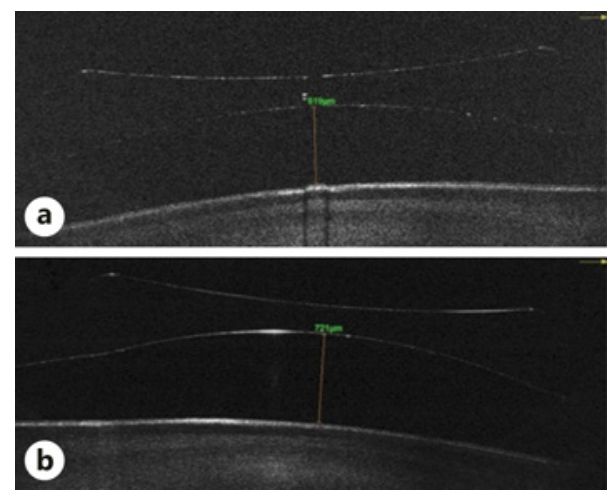

\section{Discussion}

MFS is a heritable collagen vascular disease wherein $50 \%$ patients are diagnosed as part of the evaluation for ophthalmic complaints $[7,8]$. MFS is the most common cause of heritable ectopia lentis and in fact is the most frequent ocular manifestation of MFS, occurring in approximately $75 \%$ of patients [9]. Coloboma associated with MFS is rather a rare entity but has been reported earlier in literature [10-12]. We diagnosed the patient as MFS based on revised Ghent criteria [13].

Myopia in patients with MFS is most commonly due to long axial length [14]. They may also suffer from astigmatism with both lenticular and corneal components. Laser refractive surgeries are not recommended for most MFS patients because of unpredictable outcomes owing to high lenticular astigmatism and the cornea's healing ability. Moreover, it might also interfere with future intraocular lens power calculation. To the best of our knowledge, there is no case report or case series on laser refractive surgeries in MFS.

Intraocular refractive procedures can be a viable alternative for refractive correction in MFS, providing a wider range of treatment for ametropia, more stable refraction, and better visual quality $[15,16]$. Two basic intraocular refractive procedures exist: pIOL implantation and clear lens extraction with lens implantation, also called RLE. The latter accounts for less than $1 \%$ of refractive procedures done worldwide [17], and it never got very popular as it is thought to increase the risk for retinal detachment [18] in myopic pre-presbyopic patients, is irreversible, and causes loss of accommodation. The zonular and lens instability due to coloboma/subluxation of lens is frequently complicated by the loss of capsular bag, vitreous disturbance, and endothelial cell damage. IOL power calculation is also an important issue in patients with the MFS due to longer axial length and presence of posterior staphyloma. So unless there is anterior/posterior dislocation of the lens, lens-induced glaucoma/uveitis, or inadequate visual acuity not corrected by refraction (glasses/contact lenses), RLE is generally avoided in patients with MFS.

Toric pIOLs are known to give excellent results in corneal astigmatism secondary to keratoconus [19] and post-corneal transplants [20]. To the best of our knowledge, toric pIOL implantation has never been described in literature in MFS with lens coloboma. As the patient had good anterior chamber depth, clear cornea and motivation for spectacle independence, we decided to go ahead with bilateral toric pIOL surgery which was uneventful. We understand that the possibility of pIOL rotation due to excess space in the sulcus is a possibility and needs to be considered. The incidence of retinal detachment in MFS ranges from 5 to $25.6 \%$ [21]. Cataract surgery is usually considered a safe and effective method to remove subluxated lens, but retinal detachment is also a possibility after lens extraction in MFS [22]. The vitreolensectomy surgery in MFS patients has post-operative incidence of retinal detachment rate

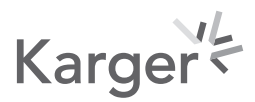


of $6 \%$ [23]. This emphasizes the importance of long-term follow-up in MFS cases. MFS patients should be routinely followed up with retinal periphery examination at each visit. Patients should also be educated regarding retinal detachment symptoms and advised to seek instant consultation in case they notice floaters, flashes, or loss of vision. In our case, the patient has been followed up for 18 months and no post-operative complications were noted.

\section{Conclusions}

Our case study is unique as it reports MFS with associated coloboma, and it is the first of its kind to attempt pIOL in such a case as no established guidelines exist for the same. A larger study population and a longer follow-up period are required to form guidelines for the same.

\section{Statement of Ethics}

This report followed guidelines to be HIPAA compliant and permission was obtained from the patient to publish identifiable photographs. The study adhered to the tenets of the Declaration of Helsinki.

\section{Conflict of Interest Statement}

None of the authors have a proprietary interest in this study or any conflicts of interest to disclose.

\section{Funding Sources}

No funding sources.

\section{Author Contributions}

Suchit Dadia: data and figure compiling. Sourabh Sharma: case report writing. Bhupesh Singh: case report writing and editing. Neha Bharti: editing and proof reading. Sudhank Bharti: idea, surgery, and final version of case report approval.

\section{Patient Consent}

The patient consented to publication of the case in writing. This report does not contain any personal information that could lead to the identification of the patient.

\section{Karger'}




\section{References}

1 Dietz HC, Cutting GR, Pyeritz RE, Maslen CL, Sakai LY, Corson GM, et al. Marfan syndrome caused by a recurrent de novo missense mutation in the fibrillin gene. Nature. 1991;352(6333):337-9.

2 Wheatley HM, Traboulsi EI, Flowers BE, Maumenee IH, Azar D, Pyeritz RE, et al. Immunohistochemical localization of fibrillin in human ocular tissues. Relevance to the Marfan syndrome. Arch Ophthalmol. 1995;113(1): 103-9.

3 Michael K, Sarah W, Iris SK, Nathalie FA, Maumenee IH, Marilyn BM. Biometry characteristics in adults and children with Marfan syndrome: from the Marfan eye consortium of Chicago. Am J Ophthalmol. 2017;177: 144-9.

4 Barsam A, Allan BD. Excimer laser refractive surgery versus phakic intraocular lenses for the correction of moderate to high myopia. Cochrane Database Syst Rev. 2012;1:CD007679.

5 Karimian F, Baradaran-Rafii A, Hashemian SJ, Hashemloo A, Jafari ME, Yaseri M, et al. Comparison of three phakic intraocular lenses for correction of myopia. J Ophthalmic Vis Res. 2014 Oct-Dec;9(4):427-33.

6 Lin H, Yan P, Yu K, Luo L, Chen J, Lin Z, et al. Anterior segment variations after posterior chamber phakic intraocular lens implantation in myopic eyes. J Cataract Refract Surg. 2013 May;39(5):730-8.

7 Mayo Clinic Staff. Mixed connective tissue disease. 2015 Apr 3.

8 Strider D, Moore T. Marfan's syndrome: a family affair. J Vasc Nurs. 1997;14.

9 Wakita M, Ryu E, Nakayasu K, Kashima K, Fujiki K, Kato K, et al. [Statistical analysis of Marfan's syndrome]. Nippon Ganka Gakkai Zasshi. 1989;93(6):682-90.

10 Thapa BB, Singh R, Ram J, Kumar A. Lens coloboma in one eye and ectopia lentis in the other eye of a patient with Marfan syndrome. BMJ Case Rep. 2014;2014:bcr2014207112.

11 Angra SK, Gupta S, Dada VK, Gupta AK. Coloboma of lens. Indian J Ophthalmol. 1984;32(1):21-2.

12 Mehrotra AS, Solanki N, Sabharwal KK. Bilateral coloboma of lens in Marfan's syndrome. Indian J Ophthalmol. 1985;33(3):201-2.

13 Loeys BL, Dietz HC, Braverman AC, Callewaert BL, De Backer J, Devereux RB, et al. The revised Ghent nosology for the Marfan syndrome. J Med Genet. 2010;47(7):476-85.

14 Maumenee IH. The eye in the Marfan syndrome. Trans Am Ophthalmol Soc. 1981;79:684-733.

15 Rosario C, Jaime B. LASIK outcomes in patients with underlying systemic contraindications: a preliminary study. Ophthalmology. 2006;113:1118-24.

16 Sanders DR. Matched population comparison of the Visian implantable collamer lens and standard LASIK for myopia of -3 to -7.88 diopters. J Refract Surg. 2007;23:537-53.

17 Bashour M. Refractive lens exchange (clear lens extraction) for Myopia correction. Ophthalmology. 2017 Dec.

18 Colin J, Robinet A, Cochener B. Retinal detachment after clear lens extraction for high myopia: seven-year follow-up. Ophthalmology. 1999 Dec;106(12):2281-5.

19 Komatsu M, Nakamura A. 3-year follow-up of posterior chamber toric phakic iol implantation for the correction of high myopic astigmatism in eyes with keratoconus. Br J Ophthalmol. 2015;99(2):177-83.

20 Poo-López A, Ferrer-Blasco T. Posterior chamber phakic intraocular lenses after penetrating keratoplasty. J Cataract Refract Surg. 2009;35:1166-73.

21 Miraldi Utz V, Coussa RG, Traboulsi EI. Surgical management of lens subluxation in Marfan syndrome. J AAPOS. 2014;18(2):140-6.

22 Wu-Chen WY, Letson RD, Summers CG. Functional and structural outcomes following lensectomy for ectopia lentis. J AAPOS. 2005;9(4):353-7.

23 Hubbard AD, Charteris DG, Cooling RJ. Vitreolensectomy in Marfan's syndrome. Eye. 1998;12(Pt 3a):412-6. 